\title{
Lattice Boltzmann Simulation of Bubble Flows
}

\author{
Takaji Inamuro, Takeshi Ogata, and Fumimaru Ogino \\ Department of Chemical Engineering, Kyoto University, Kyoto 606-8501, Japan, \\ inamuro@cheme.kyoto-u.ac.jp
}

\begin{abstract}
A lattice Boltzmann method for two-phase immiscible fluids with large density ratios is proposed. The difficulty in the treatment of large density ratio is resolved by using the projection method. The method can simulate two-phase fluid flows with the density ratio up to 1000. The method is applied to the simulations of a single rising bubble in liquid and many bubbles rising in a square duct. The terminal shapes and the terminal Reynolds numbers of the single bubble for various Morton and Eötvös numbers are in good agreement with available experimental data. The complicated unsteady structures of the interface and the flow field are illustrated in many bubbles rising in a square duct.
\end{abstract}

\section{Introduction}

Recently, the lattice Boltzmann method (LBM) has been developed into an alternative and promising numerical scheme for simulating multicomponent fluid flows [1-4]. Inamuro et al. [5, 6] have also proposed a lattice Boltzmann method for multicomponent immiscible fluids with the same density. The LBM has great advantages over conventional methods for multiphase flows. It does not track interfaces, but can maintain sharp interfaces without any artificial treatments. Also, the LBM is accurate for the mass conservation of each component fluid. Although the LBM is a promising method for multicomponent fluid flows, one of disadvantages is that all above schemes are limited to small density ratios less than 10. Usually the density ratio of liquid-gas systems is larger than 100, e.g., the density ratio of water to air is about 1000 to 1 .

The aims of the present paper are to propose an LBM for two-phase fluids with large density ratios and to apply the method to the simulations of rising bubble flows. The difficulty in the treatment of large density ratio is resolved by using the projection method [7]. Two particle velocity distribution functions are used. One is used for the calculation of an order parameter which represents two phases, and the other is used for the calculation of a predicted velocity of the two-phase fluid without a pressure gradient. The current velocity can be obtained by using the relation between the velocity and the pressure correction which is determined by solving the Poisson equation.

The method is applied to the simulations of a single rising bubble in liquid and many bubbles rising in a square duct. The terminal shapes of the single bubble for various Morton and Eötvös numbers are calculated, and the shape regime and the terminal Reynolds number are compared with available experimental 
data. The complicated structures of the interface and the flow field of many bubbles rising in a square duct are calculated.

\section{Numerical Method}

Non-dimensional variables, which are defined by using a characteristic length $L$, a characteristic particle speed $c$, a characteristic time scale $t_{0}=L / U$ where $U$ is a characteristic flow speed, and a reference density $\rho_{0}$, are used as in [8]. In the LBM, a modeled fluid, composed of identical particles whose velocities are restricted to a finite set of $N$ vectors $\boldsymbol{c}_{i}(i=1,2, \cdots, N)$, is considered. The fifteen-velocity model $(N=15)$ is used in the present paper. The velocity vectors of this model are given by

$$
\begin{aligned}
& {\left[\boldsymbol{c}_{1}, \boldsymbol{c}_{2}, \boldsymbol{c}_{3}, \boldsymbol{c}_{4}, \boldsymbol{c}_{5}, \boldsymbol{c}_{6}, \boldsymbol{c}_{7}, \boldsymbol{c}_{8}, \boldsymbol{c}_{9}, \boldsymbol{c}_{10}, \boldsymbol{c}_{11}, \boldsymbol{c}_{12}, \boldsymbol{c}_{13}, \boldsymbol{c}_{14}, \boldsymbol{c}_{15}\right]=}
\end{aligned}
$$

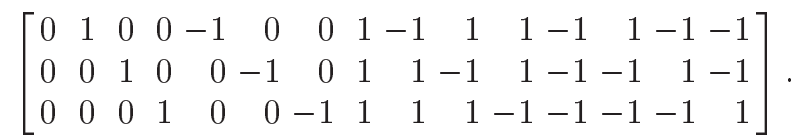

The physical space is divided into a cubic lattice, and the evolution of particle population at each lattice site is computed. Two particle velocity distribution functions, $f_{i}$ and $g_{i}$, are used. The function $f_{i}$ is used for the calculation of an order parameter which represents two phases, and the function $g_{i}$ is used for the calculation of a predicted velocity of the two-phase fluid without a pressure gradient. The evolution of the particle distribution functions $f_{i}(\boldsymbol{x}, t)$ and $g_{i}(\boldsymbol{x}, t)$ with velocity $\boldsymbol{c}_{i}$ at the point $\boldsymbol{x}$ and at time $t$ is computed by the following equations:

$$
\begin{aligned}
f_{i}\left(\boldsymbol{x}+\boldsymbol{c}_{i} \Delta x, t+\Delta t\right) & =f_{i}(\boldsymbol{x}, t)-\frac{1}{\tau_{f}}\left[f_{i}(\boldsymbol{x}, t)-f_{i}^{\mathrm{eq}}(\boldsymbol{x}, t)\right] \\
g_{i}\left(\boldsymbol{x}+\boldsymbol{c}_{i} \Delta x, t+\Delta t\right) & =g_{i}(\boldsymbol{x}, t)-\frac{1}{\tau_{g}}\left[g_{i}(\boldsymbol{x}, t)-g_{i}^{\mathrm{eq}}(\boldsymbol{x}, t)\right] \\
& -3 E_{i} c_{i z}\left(1-\frac{\rho_{\mathrm{L}}}{\rho}\right) g \Delta x+3 E_{i} c_{i \alpha} \frac{1}{\rho}\left[\frac{\partial}{\partial x_{\beta}} \mu\left(\frac{\partial u_{\beta}}{\partial x_{\alpha}}+\frac{\partial u_{\alpha}}{\partial x_{\beta}}\right)\right] \Delta x
\end{aligned}
$$

where $f_{i}^{\text {eq }}$ and $g_{i}^{\text {eq }}$ are equilibrium distribution functions, $\tau_{f}$ and $\tau_{g}$ are dimensionless single relaxation times, $\Delta x$ is a spacing of the cubic lattice, $\Delta t$ is a time step during which the particles travel the lattice spacing, $g$ is the gravitational acceleration, and the other variables, $\rho, \rho_{\mathrm{L}}, \mu$, and $\boldsymbol{u}$, and constants $E_{i}$ are defined below.

The order parameter $\phi$ distinguishing the two phases and the predicted velocity $\boldsymbol{u}^{*}$ of the multicomponent fluids are defined in terms of the two particle velocity distribution functions as follows:

$$
\phi=\sum_{i=1}^{15} f_{i}
$$




$$
\boldsymbol{u}^{*}=\sum_{i=1}^{15} g_{i} \boldsymbol{c}_{i}
$$

The equilibrium distribution functions in Eqs. (2) and (3) are given by

$$
\begin{aligned}
f_{i}^{\mathrm{eq}}=H_{i} \phi+F_{i}\left[p_{0}-\kappa_{f} \phi \nabla^{2} \phi-\right. & \left.\frac{\kappa_{f}}{6}|\nabla \phi|^{2}\right] \\
& +3 E_{i} \phi c_{i \alpha} u_{\alpha}+E_{i} \kappa_{f} G_{\alpha \beta}(\phi) c_{i \alpha} c_{i \beta}, \\
g_{i}^{\mathrm{eq}}=E_{i}\left[1+3 c_{i \alpha} u_{\alpha}-\frac{3}{2} u_{\alpha} u_{\alpha}+\right. & \frac{9}{2} c_{i \alpha} c_{i \beta} u_{\alpha} u_{\beta} \\
+ & \left.\frac{3}{2}\left(\tau_{g}-\frac{1}{2}\right) \Delta x\left(\frac{\partial u_{\beta}}{\partial x_{\alpha}}+\frac{\partial u_{\alpha}}{\partial x_{\beta}}\right) c_{i \alpha} c_{i \beta}\right] \\
& +E_{i} \frac{\kappa_{g}}{\rho} G_{\alpha \beta}(\rho) c_{i \alpha} c_{i \beta}-\frac{2}{3} F_{i} \frac{\kappa_{g}}{\rho}|\nabla \rho|^{2},
\end{aligned}
$$

where

$$
\begin{aligned}
& E_{1}=2 / 9, E_{2}=E_{3}=E_{4}=\cdots=E_{7}=1 / 9, \\
& E_{8}=E_{9}=E_{10}=\cdots=E_{15}=1 / 72, \\
& H_{1}=1, H_{2}=H_{3}=H_{4}=\cdots=H_{15}=0, \\
& F_{1}=-7 / 3, F_{i}=3 E_{i}(i=2,3,4, \cdots, 15),
\end{aligned}
$$

and

$$
G_{\alpha \beta}(\phi)=\frac{9}{2} \frac{\partial \phi}{\partial x_{\alpha}} \frac{\partial \phi}{\partial x_{\beta}}-\frac{3}{2} \frac{\partial \phi}{\partial x_{\gamma}} \frac{\partial \phi}{\partial x_{\gamma}} \delta_{\alpha \beta},
$$

with $\alpha, \beta, \gamma=x, y, z$ (subscripts $\alpha, \beta$, and $\gamma$ represent Cartesian coordinates and the summation convention is used). In the above equations, $\delta_{\alpha \beta}$ is the Kronecker delta, $\kappa_{f}$ is a constant parameter determining the width of the interface, and $\kappa_{g}$ is a constant parameter determining the strength of the surface tension. In Eq. (6), $p_{0}$ is given by

$$
p_{0}=\phi T \frac{1}{1-b \phi}-a \phi^{2}
$$

where $a, b$, and $T$ are free parameters determining the maximum and minimum values of the order parameter $\phi$. It is noted that $f_{i}$ is the same as that of the Swift et al. model [3]. The following finite-difference approximations are used to calculate the derivatives in Eqs. (6), (7), and (9):

$$
\begin{aligned}
& \frac{\partial \psi}{\partial x_{\alpha}} \approx \frac{1}{10 \Delta x} \sum_{i=1}^{15} c_{i \alpha} \psi\left(\boldsymbol{x}+\boldsymbol{c}_{i} \Delta x\right) \\
& \nabla^{2} \psi \approx \frac{1}{5 \Delta x}\left[\sum_{i=2}^{15} \psi\left(\boldsymbol{x}+\boldsymbol{c}_{i} \Delta x\right)-14 \psi(\boldsymbol{x})\right]
\end{aligned}
$$


The density in the interface is obtained by using the cut-off values of the order parameter, $\phi_{\mathrm{L}}^{*}$ and $\phi_{\mathrm{G}}^{*}$, for the liquid and gas phases with the following relation:

$$
\rho=\left\{\begin{array}{lc}
\rho_{\mathrm{G}}, & \phi<\phi_{\mathrm{G}}^{*}, \\
\frac{\Delta \rho}{2}\left[\sin \left(\frac{\phi-\overline{\phi^{*}}}{\Delta \phi^{*}} \pi\right)+1\right]+\rho_{\mathrm{G}}, \phi_{\mathrm{G}}^{*} \leq \phi \leq \phi_{\mathrm{L}}^{*}, \\
\rho_{\mathrm{L}}, & \phi>\phi_{\mathrm{L}}^{*},
\end{array}\right.
$$

where $\rho_{\mathrm{G}}$ and $\rho_{\mathrm{L}}$ are the density of gas and liquid phase, respectively, $\Delta \rho=$ $\rho_{\mathrm{L}}-\rho_{\mathrm{G}}, \Delta \phi^{*}=\phi_{\mathrm{L}}^{*}-\phi_{\mathrm{G}}^{*}$, and $\overline{\phi^{*}}=\left(\phi_{\mathrm{L}}^{*}+\phi_{\mathrm{G}}^{*}\right) / 2$. The viscosity $\mu$ in the interface is obtained by

$$
\mu=\frac{\rho-\rho_{\mathrm{G}}}{\rho_{\mathrm{L}}-\rho_{\mathrm{G}}}\left(\mu_{\mathrm{L}}-\mu_{\mathrm{G}}\right)+\mu_{\mathrm{G}},
$$

where $\mu_{\mathrm{G}}$ and $\mu_{\mathrm{L}}$ are the viscosity of gas and liquid phase, respectively. The surface tension $\sigma$ is given by

$$
\sigma=\kappa_{g} \int_{-\infty}^{\infty}\left(\frac{\partial \rho}{\partial \xi}\right)^{2} d \xi
$$

with $\xi$ being the coordinate normal to the interface [9].

Since $\boldsymbol{u}^{*}$ is not divergence free $\left(\nabla \cdot \boldsymbol{u}^{*} \neq 0\right)$, the correction of $\boldsymbol{u}^{*}$ is required. The current velocity $\boldsymbol{u}$ which satisfies the continuity equation $(\nabla \cdot \boldsymbol{u}=0)$ can be obtained by using the following equations:

$$
\begin{gathered}
\operatorname{Sh} \frac{\boldsymbol{u}-\boldsymbol{u}^{*}}{\Delta t}=-\frac{\nabla p}{\rho}, \\
\nabla \cdot\left(\frac{\nabla p}{\rho}\right)=\operatorname{Sh} \frac{\nabla \cdot \boldsymbol{u}^{*}}{\Delta t},
\end{gathered}
$$

where $\mathrm{Sh}=U / c$ is the Strouhal number and $p$ is the pressure. The Poisson equation (17) can be solved by various methods. In the present paper, we solve Eq. (17) in the framework of LBM. Namely, the following evolution equation of the velocity distribution function $h_{i}$ is used for the calculation of the pressure $p$ :

$$
h_{i}^{n+1}\left(\boldsymbol{x}+\boldsymbol{c}_{i} \Delta x\right)=h_{i}^{n}(\boldsymbol{x})-\frac{1}{\tau_{h}}\left[h_{i}^{n}(\boldsymbol{x})-E_{i} p^{n}(\boldsymbol{x})\right]-\frac{1}{3} E_{i} \frac{\partial u_{\alpha}^{*}}{\partial x_{\alpha}} \Delta x,
$$

where $n$ is the number of iterations and the relaxation time $\tau_{h}$ is given by

$$
\tau_{h}=\frac{1}{\rho}+\frac{1}{2}
$$

The pressure is obtained by

$$
p=\sum_{i=1}^{15} h_{i} .
$$




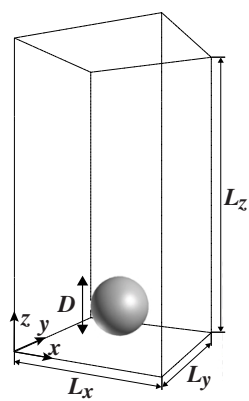

(a)

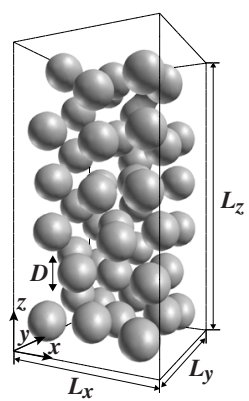

(b)

Fig. 1. Computational domain: (a) a single rising bubble, (b) 48 bubbles in a duct.
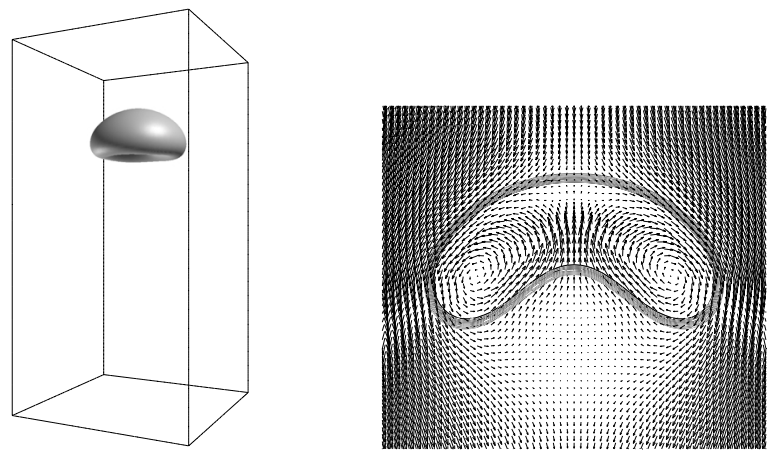

Fig. 2. Shape of a bubble and velocity vectors on $y=L_{y} / 2$ at terminal velocity for $\mathrm{M}=1, \mathrm{E}=15$ with $\rho_{\mathrm{L}} / \rho_{\mathrm{G}}=1000$.

The iteration of Eq. (18) is repeated until $\left|p^{n+1}-p^{n}\right| / \rho<\varepsilon$ is satisfied in the whole domain.

Applying the asymptotic theory to Eqs. (2), (3), and (18), we find that the asymptotic expansions of macroscopic variables, $\phi, \rho, \boldsymbol{u}$, and $p$, satisfy the phasefield advection-diffusion equation (the Cahn-Hilliard equation plus advection) for $\phi$, the continuity equation, and the Navier-Stokes equations for incompressible two-phase fluid with relative errors of $O\left[(\Delta x)^{2}\right][10]$.

It is also found in preliminary calculations that using the present method we can simulate multiphase flows with the density ratio up to 1000 .

\section{Results and Discussion}

\subsection{A Single Rising Bubble}

A single bubble with diameter $D$ is placed in a liquid inside a rectangular domain (see Fig. 1(a)) and is released at time $t=0$. The density ratio of the liquid to the 


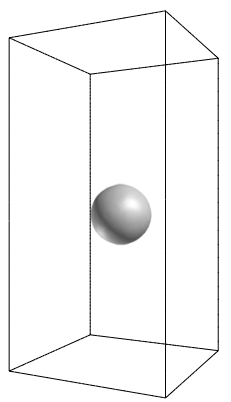

(a)

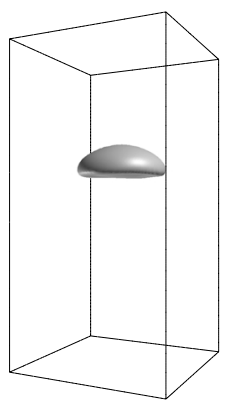

(d)

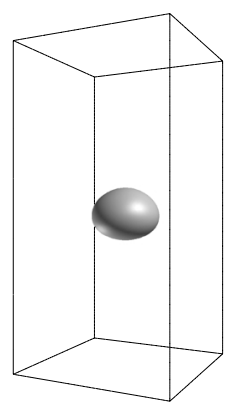

(b)

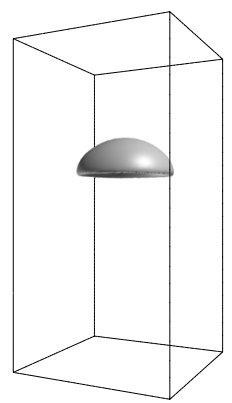

(e)

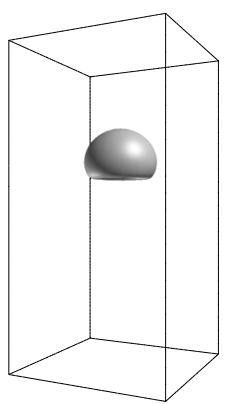

(c)

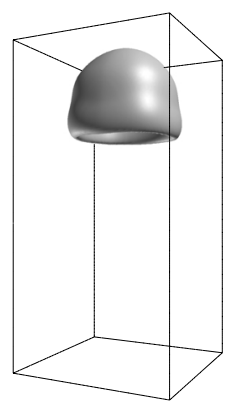

(f)

Fig. 3. Shape of a bubble at terminal velocity with $\rho_{\mathrm{L}} / \rho_{\mathrm{G}}=50$. (a) $\mathrm{M}=1 \times 10^{-3}$, $\mathrm{E}=1$; (b) $\mathrm{M}=1 \times 10^{-3}, \mathrm{E}=5$; (c) $\mathrm{M}=266, \mathrm{E}=116$; (d) $\mathrm{M}=1 \times 10^{-4}, \mathrm{E}=20$; (e) $\mathrm{M}=1 \times 10^{-3}, \mathrm{E}=42$; (f) $\mathrm{M}=43, \mathrm{E}=339$.

gas is $\rho_{\mathrm{L}} / \rho_{\mathrm{G}}=50$ or $1000\left(\rho_{\mathrm{L}}=50\right.$ or $\left.1000, \rho_{\mathrm{G}}=1\right)$. The dimensionless parameters for this phenomenon are the Morton number $\mathrm{M}=g \mu_{\mathrm{L}}^{4}\left(\rho_{\mathrm{L}}-\rho_{\mathrm{G}}\right) / \rho_{\mathrm{L}}^{2} \sigma^{3}$, the Eötvös number $\mathrm{E}=g\left(\rho_{\mathrm{L}}-\rho_{\mathrm{G}}\right) D^{2} / \sigma$, and the Reynolds number $\operatorname{Re}=\rho_{\mathrm{L}} D V / \mu_{\mathrm{L}}$ where $V$ is a terminal velocity of the bubble. The periodic boundary condition is used on all the sides of the domain. The domain is divided into an $80 \times 80 \times 160$ cubic lattice. The parameters in Eq. (10) are $a=1, b=1$, and $T=2.93 \times 10^{-1}$; it follows that the maximum and minimum values of the order parameter are $\phi_{\max }=4.031 \times 10^{-1}$ and $\phi_{\min }=2.638 \times 10^{-1}$. The cut-off values of the order parameter are $\phi_{\mathrm{L}}^{*}=3.80 \times 10^{-1}$ and $\phi_{\mathrm{G}}^{*}=2.75 \times 10^{-1}$. The other parameters are fixed at $\tau_{f}=1, \tau_{g}=1, \varepsilon=10^{-6}, D=30 \Delta x, \mu_{\mathrm{L}} / \mu_{\mathrm{G}}=50, \kappa_{f}=0.05(\Delta x)^{2}$ and $\kappa_{g}=1 \times 10^{-5}(\Delta x)^{2}$ for $\rho_{\mathrm{L}}=50$, and $\kappa_{f}=0.1(\Delta x)^{2}$ and $\kappa_{g}=1 \times 10^{-7}(\Delta x)^{2}$ for $\rho_{\mathrm{L}}=1000$. The liquid viscosity $\mu_{\mathrm{L}}$ and the gravitational acceleration $g$ are changed for various values of the Morton and Eötvös numbers.

Figure 2 shows the terminal shape of a bubble and the velocity vectors relative to the averaged bubble velocity for $\mathrm{M}=1$ and $\mathrm{E}=15$ with $\rho_{\mathrm{L}} / \rho_{\mathrm{G}}=1000$. It is seen that the bubble is deformed into a mushroom shape and a circulatory flow is induced inside the bubble. Figure 3 shows terminal shapes of a bubble for various 

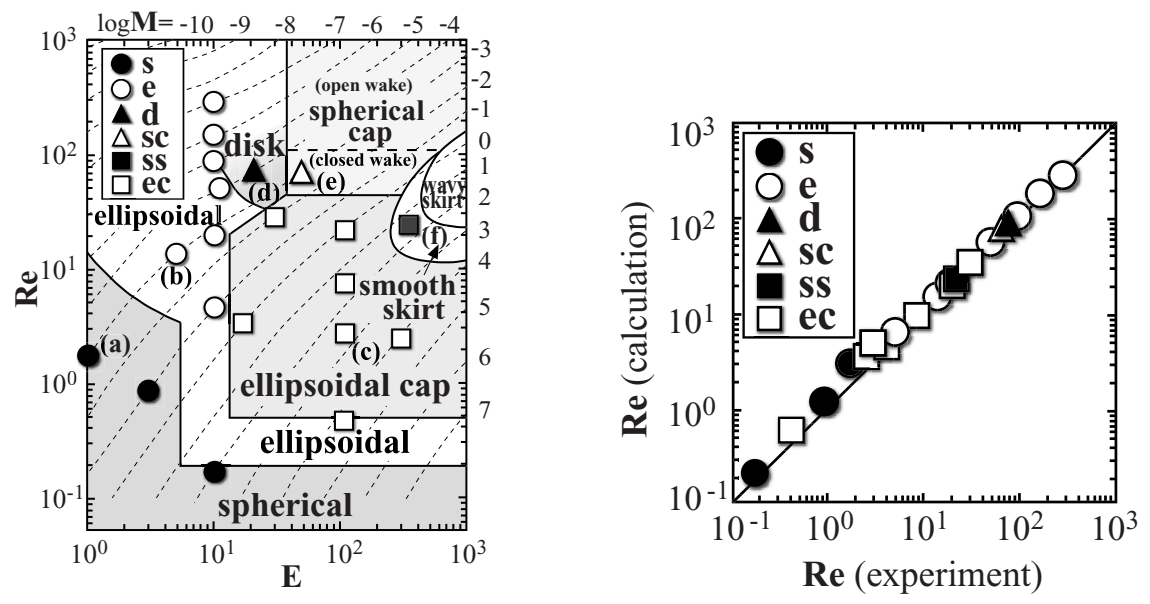

Fig. 4. Comparison of shape regime map (left) and terminal Reynolds number (right) with experimental data $[11,12]$.
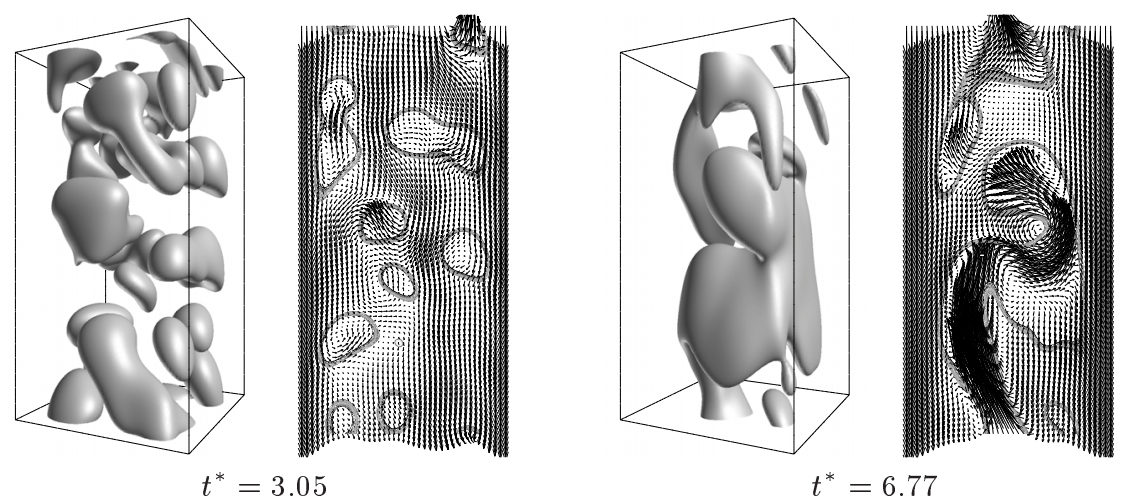

Fig. 5. Shape of interface and velocity vectors on $y=0.425 L_{y}$ of 48 bubbles for $\mathrm{M}=$ $1 \times 10^{-3}$ and $\mathrm{E}=5$ with $\rho_{\mathrm{L}} / \rho_{\mathrm{G}}=1000\left(t^{*}=t V^{*} / L_{x}\right.$ where $V^{*}$ is the averaged gas-phase velocity of the result on the right-hand side).

values of the Morton and Eötvös numbers with $\rho_{\mathrm{L}} / \rho_{\mathrm{G}}=50$. The calculated results are classified into (a) spherical, (b) ellipsoidal, (c) ellipsoidal cap, (d) disk, (e) spherical cap, and (f) smooth skirt shapes. We calculate terminal shapes and terminal Reynolds numbers for various conditions, and those are in good agreement with the experimental regime map [11] and the experimental terminal Reynolds numbers [12] as shown in Fig. 4. 


\subsection{Bubbles Rising in a Square Duct}

Many bubbles (48 bubbles) are placed in a square duct (see Fig. 1(b)) and are released at time $t=0$. The density ratio is $\rho_{\mathrm{L}} / \rho_{\mathrm{G}}=1000$. The periodic boundary condition is used on the top and bottom of the domain, and the bounce-back condition is used on the sides of the domain. The parameters of computation are the same as those used in the previous section except $D=20 \Delta x, \mu_{\mathrm{L}} \Delta x=2.506$, and $g \Delta x=2.778 \times 10^{-7}$. Figure 5 shows the calculated results for $\mathrm{M}=1 \times 10^{-3}$ and $\mathrm{E}=5$. It is seen that the bubbles coalesce each other, and then the interface between the gas and liquid phases is deformed into a complicated unsteady shape representing a churn flow. Note that the complicated flow field can be stably calculated even for the large density ratio of $\rho_{\mathrm{L}} / \rho_{\mathrm{G}}=1000$. Also, it is found that the velocity field inside the gas phase is more complicated than those in the liquid.

\section{Concluding Remarks}

A lattice Boltzmann method for two-phase immiscible fluids with large density ratios has been developed. The method can simulate two-phase flows with the density ratio up to 1000 . In the application to a single rising bubble, the calculated results are in good agreement with experimental data. For many bubbles rising in a square duct, the complicated unsteady structures of the interface and the velocity field can be stably simulated. The method is considered to be a promising method for simulating two-phase flows with large density ratios.

The accuracy of the method would depend on the interface width related to the parameter $\kappa_{f}$, the mobility determined by the parameter $\tau_{f}$, and the lattice spacing $\Delta x$. The study of the accuracy concerning these parameters is required in future work.

\section{References}

1. Gunstensen, A.K., Rothman, D.H., Zaleski, S., Zanetti, G.: Lattice Boltzmann model of immiscible fluids. Phys. Rev. A 43 (1991) 4320-4327

2. Shan, X., Chen, H.: Lattice Boltzmann model for simulating flows with multiple phases and components. Phys. Rev. E 47 (1993) 1815-1819

3. Swift, M.R., Osborn, W.R., Yeomans, J.M.: Lattice Boltzmann simulation of nonideal fluids. Phys. Rev. Lett. 75 (1995) 830-833

4. He, X., Chen, S., Zhang, R.: A lattice Boltzmann scheme for incompressible multiphase flow and its application in simulation of Rayleigh-Taylor instability. J. Comput. Phys. 152 (1999) 642-663

5. Inamuro, T., Miyahara, T., Ogino, F.: Lattice Boltzmann simulations of drop deformation and breakup in simple shear flow. Computational Fluid Dynamics 2000 (ed. N. Satofuka) (2001) 499-504

6. Inamuro, T., Tomita, R., Ogino, F.: Lattice Boltzmann simulations of drop deformation and breakup in shear flows. Int. J. Mod. Phys. B (2002) in press.

7. Chorin, A.J.: Numerical solution of the Navier-Stokes equations. Math. Comput. 22 (1968) 745-762 
8. Inamuro, T., Yoshino, M., Ogino, F.: Accuracy of the lattice Boltzmann method for small Knudsen number with finite Reynolds number. Phys. Fluids 9 (1997) 3535-3542

9. Inamuro, T., Konishi, N., Ogino, F.: A Galilean invariant model of the lattice Boltzmann method for multiphase fluid flows using free-energy approach. Comput. Phys. Commun. 129 (2000) 32-45

10. Inamuro, T., Ogata, T., Tajima, S., Ogino, F.: A lattice Boltzmann method for incompressible two-phase flows with large density difference. (to be submitted)

11. Bhaga, D., Weber, M.E.: Bubbles in viscous liquids: shape, wake and velocities. J. Fluid Mech. 54 (1981) 61-85

12. Grace, J.R.: Shapes and velocities of bubbles rising in infinite liquids. Trans. Inst. Chem. Eng. 51 (1973) 116-120 